\title{
MAXIMUM AND ANTIMAXIMUM PRINCIPLES FOR SOME NONLOCAL DIFFUSION OPERATORS
}

\author{
JORGE GARCÍA-MELIÁN AND JULIO D. ROSSI
}

\begin{abstract}
In this work we consider the maxiumum and antimaximum principles for the nonlocal Dirichlet problem

$$
J * u-u+\lambda u+h=\int_{\mathbb{R}^{N}} J(x-y) u(y) d y-u(x)+\lambda u(x)+h(x)=0
$$

in a bounded domain $\Omega$, with $u(x)=0$ in $\mathbb{R}^{N} \backslash \Omega$. The kernel $J$ in the convolution is assumed to be a continuous, compactly supported nonnegative function with unit integral. We prove that for $\lambda<\lambda_{1}(\Omega)$, the solution verifies $u>0$ in $\bar{\Omega}$ if $h \in L^{2}(\Omega), h \geq 0$, while for $\lambda>$ $\lambda_{1}(\Omega)$, and $\lambda$ close to $\lambda_{1}(\Omega)$, the solution verifies $u<0$ in $\bar{\Omega}$, provided $\int_{\Omega} h(x) \phi(x) d x>0, h \in L^{\infty}(\Omega)$. This last assumption is also shown to be optimal. The "Neumann" version of the problem is also analyzed.
\end{abstract}

\section{INTRODUCTION}

This note is mainly concerned with the validity of the maximum and antimaximum principles for the nonlocal inhomogeneous linear problem

$$
\left\{\begin{array}{cl}
\int_{\mathbb{R}^{N}} J(x-y) u(y) d y-u(x)+\lambda u(x)+h(x)=0 & x \in \Omega \\
u(x)=0 & x \in \mathbb{R}^{N} \backslash \Omega .
\end{array}\right.
$$

Here $\Omega$ is a bounded domain of $\mathbb{R}^{N}, h$ is a given function and $\lambda$ is a real parameter. The operator $J * u-u=\int_{\mathbb{R}^{N}} J(x-y) u(y) d y-u(x)$ is a nonlocal diffusion operator that has been recently used to model several physical situations (see for example [23] in the context of biological models). Without further mention, we are always assuming that the kernel $J$ is continuous, nonnegative, supported in the unit ball $B$ of $\mathbb{R}^{N}$ and with unit integral. We also suppose that $J>0$ in $B$ and that $J(-x)=J(x)$ for every $x$. The condition $u(x)=0$ in $\mathbb{R}^{N} \backslash \Omega$ is the nonlocal analogue to the usual Dirichlet boundary condition $\left.u\right|_{\partial \Omega}=0$ imposed when one considers the usual Laplacian as the diffusion operator, see [9].

The general problem

$$
\left\{\begin{array}{cl}
(J * u)(x)-u(x)=f(x, u(x)) & x \in \Omega, \\
u(x)=0 & x \in \mathbb{R}^{N} \backslash \Omega,
\end{array}\right.
$$

and its parabolic version have been widely treated in the recent literature. In most of the references, $\Omega=\mathbb{R}^{N}$ and that the Dirichlet condition is not present. We quote for instance [5], [7], [8], [10], [18], [19], [21], [22] and [32], devoted to travelling front type solutions to the parabolic problem when $\Omega=\mathbb{R}$, and [6], [11], [12], [20], [31], that study problem (1.2) with a logistic type, bistable or power-like nonlinearity. The particular instance of the 
parabolic problem in $\mathbb{R}^{N}$ when $f=0$ is considered in [9], [27], while the "Neumann" boundary condition for the same problem is treated in [1], [16] and [17]. See also [28] for the appearance of convective terms and [14], [15] for interesting features in other related nonlocal problems. We also mention the paper [26], where some logistic equations and systems of Lotka-Volterra type are studied, and interesting biological conclusions are obtained.

Problem (1.1) is the nonlocal analogous to

$$
\left\{\begin{array}{cl}
\Delta u(x)+\lambda u(x)+h(x)=0 & x \in \Omega, \\
u(x)=0 & x \in \partial \Omega .
\end{array}\right.
$$

One can see this analogy considering the functional whose critical points are solutions to (1.1) and performing a first order Taylor expansion; we refer to [6] for details.

Since the maximum and antimaximum principles have shown to be powerful tools when analyzing nonlinear elliptic problems related to (1.3), we want to analyze them in the context of the nonlocal problem (1.1).

Recall that the maximum principle is well known for problem (1.3) in the following form: if $h \geq 0$ and $\lambda<\sigma_{1}(\Omega)$, where $\sigma_{1}(\Omega)$ denotes the first eigenvalue of the Dirichlet Laplacian in $\Omega$, then $u>0$ in $\Omega$ and $\frac{\partial u}{\partial \nu}<0$ on $\partial \Omega$, where $\nu$ stands for the outward unit normal to $\partial \Omega$. Moreover, the condition $\lambda<\lambda_{1}(\Omega)$ is known to be also necessary (see [30], [33], [35] and also [25] for a version involving the $p$-Laplacian).

With respect to the antimaximum principle, it has been proved that for $h \in L^{p}(\Omega), p>N$, such that $\int_{\Omega} h(x) \psi(x) d x>0$, with $\psi>0$ an eigenfunction associated to $\sigma_{1}(\Omega)$, there exists $\delta>0$ such that $u<0$ in $\Omega$ and $\frac{\partial u}{\partial \nu}>0$ on $\partial \Omega$ for $\sigma_{1}(\Omega)<\lambda<\sigma_{1}(\Omega)+\delta$. See [2], [13], and also [3] for a quasilinear version. It was later proved in [34] that the condition $h \in L^{p}(\Omega), p>N$ is necessary.

Our main objective in this work is to show that a version of the maximum and antimaximum principles remains valid for problem (1.1).

A word on the notion of solution to (1.1): by a solution we mean a function $u \in L^{1}(\Omega)$ which verifies (1.1) almost everywhere. Although in most places we are dealing with $h \in L^{2}(\Omega)$, which forces $u \in L^{2}(\Omega)$. However, we remark that with $h \in L^{\infty}(\Omega)$ it always follows that $u \in L^{\infty}(\Omega)$, and we are requiring this extra regularity for the validity of the antimaximum principle. It will be also shown that this condition is not superfluous.

An important role in what follows will be played by the principal eigenvalue $\lambda_{1}(\Omega)$ of the problem

$$
\left\{\begin{array}{cl}
(J * u)(x)-u(x)+\lambda u(x)=0 & x \in \Omega, \\
u(x)=0 & x \in \mathbb{R}^{N} \backslash \Omega,
\end{array}\right.
$$

whose existence and properties will be briefly considered in Section 2. Now, we only quote that $\lambda_{1}(\Omega)$ is positive and less than one, and it has an associated eigenfunction $\phi \in C(\bar{\Omega})$ which verifies $\phi>0$ in $\bar{\Omega}$ (let us mention in passing that $\phi$ has a jump discontinuity across $\partial \Omega$, see [4]).

We now come to the statement of the maximum principle. Let us mention that problem (1.1) has a unique solution for every $h \in L^{2}(\Omega)$ provided 
$\lambda<\lambda_{1}(\Omega)$ or $\lambda \in\left(\lambda_{1}(\Omega), \lambda_{1}(\Omega)+\varepsilon\right)$, if $\varepsilon$ is small enough (see Remark 1 in Section 2).

Theorem 1. Let $h \in L^{2}(\Omega)$ be such that $h \geq 0, h \not \equiv 0$, and let $u \in L^{2}(\Omega)$ be the solution to (1.1) with $\lambda<\lambda_{1}(\Omega)$. Then $u>0$ in $\bar{\Omega}$.

We remark that the condition $u>0$ in $\bar{\Omega}$ means in this context that ess inf $u>0$, since for $h \in L^{2}(\Omega)$ we only have $u \in L^{2}(\Omega)$.

Next, we consider the antimaximum principle.

Theorem 2. Let $h \in L^{\infty}(\Omega)$ verify

$$
\int_{\Omega} h(x) \phi(x) d x>0 .
$$

Then there exists $\varepsilon=\varepsilon(h)>0$ such that for $\lambda \in\left(\lambda_{1}(\Omega), \lambda_{1}(\Omega)+\varepsilon\right)$ the unique solution to (1.1) verifies $u<0$ in $\bar{\Omega}$.

We also consider the question of optimality of the hypothesis $h \in L^{\infty}(\Omega)$. We stress that if $h \notin L^{\infty}(\Omega)$ then $u \notin L^{\infty}(\Omega)$, and this provokes the failure of the antimaximum principle.

Theorem 3. For every small $\varepsilon>0$ and $\lambda \in\left(\lambda_{1}(\Omega), \lambda_{1}(\Omega)+\varepsilon\right)$, there exists $h \in L^{2}(\Omega), h \notin L^{\infty}(\Omega)$ with

$$
\int_{\Omega} h(x) \phi(x) d x>0
$$

such that the unique solution to (1.1) is positive somewhere in $\Omega$.

We can also consider the "Neumann" boundary condition, that is, the problem

$$
\int_{\Omega} J(x-y) u(y) d y-u(x)+\lambda u(x)+h(x)+\int_{\mathbb{R}^{N} \backslash \Omega} J(x-y) g(y) d y=0,
$$

for $x \in \Omega$. Here $h, g \in L^{2}(\Omega)$, and the term

$$
\int_{\mathbb{R}^{N} \backslash \Omega} J(x-y) g(y) d y
$$

is the nonlocal counterpart of the nonhomogeneous Neumann boundary condition $\partial u / \partial \eta=g$ (see [9], [16] and [17]).

The only significant difference of the results we obtain for problem (1.5) with respect to the previous ones is that the principal eigenvalue is now $\widetilde{\lambda}_{1}(\Omega)=0$, with a constant eigenfunction.

Theorem 4. Let $h, g \in L^{2}(\Omega)$ be such that $h, g \geq 0,(h, g) \not \equiv(0,0)$, and let $u \in L^{2}(\Omega)$ be the solution to (1.5) with $\lambda<\lambda_{1}(\Omega)$. Then $u>0$ in $\bar{\Omega}$.

Theorem 5. Let $h \in L^{\infty}(\Omega), g \in L^{2}(\Omega)$ verify

$$
\int_{\Omega} h(x) d x+\int_{\Omega}\left(\int_{\mathbb{R}^{N} \backslash \Omega} J(x-y) g(y) d y\right) d x>0 .
$$

Then there exists $\varepsilon=\varepsilon(h, g)>0$ such that for $\lambda \in(0, \varepsilon)$ the unique solution to (1.5) verifies $u<0$ in $\bar{\Omega}$. 
Like for the Dirichlet problem, the condition $h \in L^{\infty}(\Omega)$ is essential for the validity of the antimaximum principle. For simplicity, we state its failure only for $g=0$.

Theorem 6. For every small $\varepsilon>0$ and $\lambda \in(0, \varepsilon)$, there exists $h \in L^{2}(\Omega)$, $h \notin L^{\infty}(\Omega)$ such that

$$
\int_{\Omega} h(x) d x>0
$$

and the unique solution to (1.5) is positive somewhere in $\Omega$.

The paper is organized as follows: in Section 2 we state and prove some auxiliary results which deal with a different version of the maximum principle and the principal eigenvalue for the nonlocal operator. In Section 3 we prove the theorems: only Theorems 1,2 and 3 will be proved, since the arguments used to show Theorems 4,5 and 6 are completely analogous.

\section{Preliminaries}

In this section we gather some results which are important to understand the maximum and antimaximum principles: the validity of the maximum principle in a slightly different form than the one considered in the introduction and the existence of a principal eigenvalue of problem (1.4).

We begin with a form of the maximum principle which will be used in the sequel. We introduce the operator:

$$
L_{M} u(x)=(J * u)(x)-(1+M) u(x),
$$

with $M \geq 0$. The validity of the maximum principle for $L_{M}$ is well-known, but we include a proof for completeness (see [18], [19]; and also [26] for a parabolic version).

Theorem 7. Let $u \in L^{2}\left(\mathbb{R}^{N}\right)$ verify $L_{M} u \leq 0$ in $\Omega$ with $u \geq 0$ in $\mathbb{R}^{N} \backslash \Omega$. Then either $u>0$ or $u \equiv 0$ in $\bar{\Omega}$.

Proof. Let $u^{-}=\min \{u, 0\}$. Note that $u^{-} \leq 0$. We have $u^{-}(x)=0$ in $\mathbb{R}^{N} \backslash \Omega$, and

$$
\begin{aligned}
& \int_{\Omega} \int_{\Omega} J(x-y) u(y) u^{-}(x) d y d x \geq \int_{\Omega}\left(\int_{\mathbb{R}^{N}} J(x-y) u(y) d y\right) u^{-}(x) d x \\
& \quad \geq(1+M) \int_{\Omega} u(x) u^{-}(x) d x .
\end{aligned}
$$

This implies

$$
\int_{\Omega} \int_{\Omega} J(x-y) u^{-}(y) u^{-}(x) d y d x-\int_{\Omega} u^{-}(x)^{2} d x \geq M \int_{\Omega} u^{-}(x)^{2} d x .
$$

However, thanks to the symmetry of $J$ and Fubini's theorem, it is easy to see that the left-hand side of (2.1) equals

$$
-\frac{1}{2} \int_{\Omega} \int_{\Omega} J(x-y)\left(u^{-}(x)-u^{-}(y)\right)^{2} d y d x
$$

and thus (2.1) implies $u^{-}=0$ in $\Omega$, that is, $u \geq 0$ in $\Omega$. 
To end the proof we argue by contradiction. Assume that ess inf $u=0$. This implies that for every $\varepsilon>0$, there exists a set $\mathcal{U}$ of positive measure such that $u(x) \leq \varepsilon$ for almost every $x \in \mathcal{U}$. Choose $x_{\varepsilon}$ such that

$$
(J * u)\left(x_{\varepsilon}\right)-(1+M) u\left(x_{\varepsilon}\right) \leq 0
$$

and

$$
u\left(x_{\varepsilon}\right) \leq \varepsilon
$$

Then

$$
\int_{\mathbb{R}^{N}} J\left(x_{\varepsilon}-y\right) u(y) d y \leq(1+M) \varepsilon .
$$

Now notice that $J(z) \geq c^{-1}>0$ for $|z|<1 / 2$, and hence

$$
\int_{B_{1 / 2}\left(x_{\varepsilon}\right)} u(y) d y \leq c(1+M) \varepsilon .
$$

If we now let $\varepsilon \rightarrow 0$, the points $x_{\varepsilon}$ will accumulate at a point $x_{0} \in \bar{\Omega}$, and it will follow thanks to (2.2) that

$$
\int_{B_{1 / 2}\left(x_{0}\right)} u(y) d y=0 .
$$

Hence, $u=0$ a.e. in $B_{1 / 2}\left(x_{0}\right)$. We can continue with this argument to prove that $u \equiv 0$ in $\Omega$.

Next, we consider the issue of existence of a principal eigenvalue for the problem considered in the introduction:

$$
\left\{\begin{array}{cl}
(J * u)(x)-u(x)+\lambda u(x)=0 & x \in \Omega, \\
u(x)=0 & x \in \mathbb{R}^{N} \backslash \Omega .
\end{array}\right.
$$

Since it is not the most important point here, we only sketch the proof. See [24] for a detailed proof (we refer also to [26] or [20] for existence of the principal eigenvalue in similar situations).

Theorem 8. Problem (2.3) admits an eigenvalue $\lambda_{1}(\Omega)$ associated to an eigenfunction $\phi \in C(\bar{\Omega})$ which is positive in $\bar{\Omega}$. Furthermore, $\lambda_{1}(\Omega)$ is simple and unique and verifies $0<\lambda_{1}(\Omega)<1$. It can be variationally characterized as

$$
\lambda_{1}(\Omega)=1-\sup _{\substack{u \in L^{2}(\Omega) \\ u \neq 0}} \frac{\int_{\Omega} \int_{\Omega} J(x-y) u(x) u(y) d y d x}{\int_{\Omega} u(x)^{2} d x} .
$$

Sketch of the proof. It is easily seen that $\lambda$ is an eigenvalue of (2.3) if and only if $\mu=1-\lambda$ is an eigenvalue of $L u=J * u$ in $L^{2}(\Omega)$. Since $L$ is self-adjoint, it follows by standard spectral theory that $\mu_{1}=\|L\|$ is an eigenvalue of $L$ (moreover notice that $L$ is positive). On the other hand, the eigenvalue problem can also be considered in $C(\bar{\Omega})$, and since $L$ is compact there, an application of the Krein-Rutman theorem (Theorem 6.2 in [29]) gives that $\mu_{1}$ possesses the stated properties. We remark that the operator $L$ is not strongly positive in the sense that, for a nonnegative and nontrivial $u \in C(\bar{\Omega})$, there exists a positive integer $n$ such that $L^{n} u>0$ in $\bar{\Omega}$. 
Remark 1. An important corollary to Theorem 7 is that if $u \in L^{2}\left(\mathbb{R}^{N}\right)$ verifies $L_{M} u=0$ in $\Omega$ with $u=0$ in $\mathbb{R}^{N} \backslash \Omega$ then $u \equiv 0$. Thus it is standard to conclude that the problem

$$
\left\{\begin{array}{cl}
(J * u)(x)-(1+M) u(x)=h(x) & x \in \Omega, \\
u(x)=0 & x \in \mathbb{R}^{N} \backslash \Omega,
\end{array}\right.
$$

admits a unique solution $u \in L^{2}\left(\mathbb{R}^{N}\right)$ for every $h \in L^{2}(\Omega)$. Therefore, it follows that the problem

$$
\left\{\begin{array}{cl}
(J * u)(x)-u(x)+\lambda u(x)=h(x) & x \in \Omega, \\
u(x)=0 & x \in \mathbb{R}^{N} \backslash \Omega,
\end{array}\right.
$$

admits a unique solution $u \in L^{2}(\Omega)$ for every $h \in L^{2}(\Omega)$ provided that $\lambda$ is not an eigenvalue of $(2.3)$.

\section{MAXIMUM AND ANTIMAXIMUM PRINCIPLES}

In this section we prove Theorems 1,2 and 3. Although it has already been pointed out, we remark that the operator $L u=J * u$ is compact in $L^{\infty}(\Omega)$, even with values in $C(\bar{\Omega})$, since $J$ is uniformly continuous. This will be an essential ingredient in most of the proofs.

Note that in all the cases considered, problem (1.1) has a unique solution thanks to Remark 1.

Proof of Theorem 1. Multiply the equation in (1.1) by $u^{-}=\min \{u, 0\}$ and integrate in $\Omega$ to obtain

$$
\int_{\Omega} \int_{\Omega} J(x-y) u(y) u^{-}(x) d y d x+(\lambda-1) \int_{\Omega} u^{-}(x)^{2} d x \geq 0
$$

which immediately implies

$$
\int_{\Omega} \int_{\Omega} J(x-y) u^{-}(y) u^{-}(x) d y d x+(\lambda-1) \int_{\Omega} u^{-}(x)^{2} d x \geq 0 .
$$

Thanks to the variational characterization (2.4), we arrive at

$$
\left(\lambda-\lambda_{1}(\Omega)\right) \int_{\Omega} u^{-}(x)^{2} d x \geq 0,
$$

and since $\lambda<\lambda_{1}(\Omega)$, we obtain $u^{-} \equiv 0$ in $\Omega$, that is, $u \geq 0$.

Finally, we choose $M>0$ large so that $\lambda+M>0$, and observe that $L_{M} u=-(\lambda+M) u-h \leq 0$. By Theorem $7, u>0$ in $\bar{\Omega}$ (the possibility $u \equiv 0$ is ruled out since $h \not \equiv 0)$. This completes the proof.

Remark 2. The condition $h \geq 0$ can be slightly relaxed if $h \in L^{\infty}(\Omega)$. A careful inspection of the proofs below shows that we can assume instead

$$
\int_{\Omega} h(x) \phi(x) d x>0
$$

where $\phi$ is a positive eigenfunction associated to $\lambda_{1}(\Omega)$, if $\lambda<\lambda_{1}(\Omega)$ is close to $\lambda_{1}(\Omega)$ (depending on $h$ ). In this case we still get $u>0$ in $\bar{\Omega}$. 
Proof of Theorem 2. We argue by contradiction. Assume that there exists a sequence $\lambda_{n} \rightarrow \lambda_{1}(\Omega), \lambda_{n}>\lambda_{1}(\Omega)$, with corresponding solutions $u_{n}$ not verifying $u_{n}<0$ in $\bar{\Omega}$. We claim that $\left|u_{n}\right|_{\infty} \rightarrow+\infty$. Assume this claim does not hold. Taking a subsequence if necessary, we may assume $\left|u_{n}\right|_{\infty} \leq C$ for a positive constant $C$. Since $L u=J * u$ is compact, it follows that $J * u_{n}$ converges uniformly in $\bar{\Omega}$, and then $\left(\lambda_{n}-1\right) u_{n}=-J * u_{n}-h$ also converges uniformly. Since $\lambda_{n}-1 \rightarrow \lambda_{1}(\Omega)-1<0$ (Theorem 8), this shows that $u_{n}$ converges uniformly in $\bar{\Omega}$ to a function $u$. Passing to the limit in (1.1), we have

$$
\left\{\begin{array}{cl}
(J * u)(x)-u(x)+\lambda_{1}(\Omega) u(x)=h(x) & x \in \Omega, \\
u(x)=0 & x \in \mathbb{R}^{N} \backslash \Omega .
\end{array}\right.
$$

Let $\phi$ be the positive eigenfunction associated to $\lambda_{1}(\Omega)$ normalized according to $|\phi|_{\infty}=1$. Multiplying by $\phi$ and integrating we get

$$
\begin{aligned}
\int_{\Omega} h(x) \phi(x) d x= & \int_{\Omega} \int_{\Omega} J(x-y) u(y) \phi(x) d y d x \\
& +\left(\lambda_{1}(\Omega)-1\right) \int_{\Omega} u(x) \phi(x) d x \\
= & \int_{\Omega} \int_{\Omega} J(x-y) \phi(x) u(y) d x d y \\
& \quad+\left(\lambda_{1}(\Omega)-1\right) \int_{\Omega} u(x) \phi(x) d x \\
= & 0,
\end{aligned}
$$

thanks to Fubini's theorem. This contradiction proves the claim, that is, $\left|u_{n}\right|_{\infty} \rightarrow \infty$. Now let $v_{n}=u_{n} /\left|u_{n}\right|_{\infty}$. Notice that $v_{n}$ satisfies

$$
\left\{\begin{array}{cl}
\left(J * v_{n}\right)(x)-v_{n}(x)+\lambda_{n} v_{n}(x)+\frac{1}{\left|u_{n}\right|_{\infty}} h(x)=0 & x \in \Omega, \\
v_{n}(x)=0 & x \in \mathbb{R}^{N} \backslash \Omega .
\end{array}\right.
$$

By the compactness of $L$ it follows that $J * v_{n}$ converges uniformly in $\bar{\Omega}$, and it is shown as before that this implies the uniform convergence of $v_{n}$ to a function $v_{0}$. This function verifies $\left|v_{0}\right|_{\infty}=1$ and

$$
\left\{\begin{array}{cl}
\left(J * v_{0}\right)(x)-v_{0}(x)+\lambda_{1}(\Omega) v_{0}(x)=0 & x \in \Omega, \\
v_{0}(x)=0 & x \in \mathbb{R}^{N} \backslash \Omega .
\end{array}\right.
$$

Thus $v_{0}= \pm \phi$ (by Theorem 8 again), and hence $u_{n}$ has a constant sign in $\bar{\Omega}$ for large $n$. Since we are assuming that $u_{n}$ is not negative, it follows that $v_{0}=\phi$, and hence $u_{n}$ is strictly positive in $\bar{\Omega}$ for large $n$.

Now we multiply the equation verified by $u_{n}$ by $\phi$ and integrate in $\Omega$, to arrive at

$$
\left(\lambda_{n}-\lambda_{1}(\Omega)\right) \int_{\Omega} u_{n}(x) \phi(x) d x=-\int_{\Omega} h(x) \phi(x) d x .
$$

Since $\lambda_{n}>\lambda_{1}(\Omega)$ and $u_{n}$ is positive for large $n$, the left-hand side of (3.1) is positive, while the right-hand side is negative by assumption. This contradiction proves the theorem. 
Proof of Theorem 3. Note that $u \in L^{\infty}(\Omega)$ implies $h=J * u-u+\lambda u \in$ $L^{\infty}(\Omega)$. Then, if $h \notin L^{\infty}(\Omega)$ we have $u \notin L^{\infty}(\Omega)$.

Fix $\lambda \in\left(\lambda_{1}(\Omega), \lambda_{1}(\Omega)+\varepsilon\right)$, for some arbitrary $\varepsilon>0$. Choose $h_{1} \in L^{\infty}(\Omega)$ with

$$
\int_{\Omega} h_{1}(x) \phi(x) d x>0
$$

and let $u_{1}$ be the corresponding unique solution to (1.1), which verifies $u_{1} \in L^{\infty}(\Omega)$.

Let $h_{2} \in L^{2}(\Omega)$ such that $h_{2} \notin L^{\infty}(\Omega)$ and let $u_{2} \in L^{2}(\Omega)$ be the solution to (1.1) with $h=h_{2}$. Since $u_{2} \notin L^{\infty}(\Omega)$, it follows that ess sup $u_{2}=+\infty$ or ess inf $u_{2}=-\infty$. Choose $\delta>0$ and let $h=h_{1} \pm \delta h_{2}$, where we take the plus sign if ess $\sup u_{2}=+\infty$ and the minus sign if ess inf $u_{2}=-\infty$. We can take $\delta$ small enough so that

$$
\int_{\Omega} h(x) \phi(x) d x=\int_{\Omega} h_{1}(x) \phi(x) d x \pm \delta \int_{\Omega} h_{2}(x) \phi(x) d x>0 .
$$

Notice that the solution to (1.1) with $h=h_{1} \pm \delta h_{2}$ is $u=u_{1} \pm \delta u_{2}$, and since ess $\sup u=+\infty$ (thanks to the appropriate choice of the sign), we have that $u$ is positive somewhere in $\bar{\Omega}$.

Acknowledgements. Partially supported by MEC and FEDER under grant MTM2005-06480 (Spain), CONICET (Argentina) and UBA X066 (Argentina).

\section{REFERENCES}

[1] F. Andreu, J. M. Mazón, J.D. Rossi, J. Toledo, The Neumann problem for nonlocal nonlinear diffusion equations, to appear in J. Evol. Eqns.

[2] D. Arcoya, J. L. GÁmez, Bifurcation theory and related problems: antimaximum principle and resonance, Comm. Partial Diff. Eqns. 26 (2001), 1879-1911.

[3] D. Arcoya, J. D. Rossi, Antimaximum principle for quasilinear problems, Adv. Diff. Eqns. 9 (2004), 1185-1200.

[4] G. Barles, E. Chasseigne, C. Imbert, On the Dirichlet problem for second-order elliptic integro-differential equations, To appear in Indiana Univ. Math. J.

[5] P. Bates, X. Chen, A. ChmaJ, Heteroclinic solutions of a van der Waals model with indefinite nonlocal interactions, Calc. Var. 24 (2005), 261-281.

[6] P. Bates, A. ChmaJ, An integrodifferential model for phase transitions: stationary solutions in higher space dimensions, J. Stat. Phys. 95 (1999), 1119-1139.

[7] P. Bates, P. Fife, X. Ren, X. WANG, Traveling waves in a convolution model for phase transitions, Arch. Rat. Mech. Anal. 138 (1997), 105-136.

[8] J. CARR, A. ChMAJ, Uniqueness of traveling waves for nonlocal monostable equations, Proc. Amer. Math. Soc. 132 (2004), 2433-2439.

[9] E. Chasseigne, M. Chaves, J.D. Rossi, Asymptotic behavior for nonlocal diffusion equations, J. Math. Pures Appl. 86 (2006), 271-291.

[10] X. Chen, Existence, uniqueness and asymptotic stability of traveling waves in nonlocal evolution equations, Adv. Diff. Eqns. 2 (1997), 125-160.

[11] A. ChmaJ, X. Ren, Homoclinic solutions of an integral equation: existence and stability, J. Diff. Eqns. 155 (1999), 17-43.

[12] A. ChmaJ, X. Ren, The nonlocal bistable equation: stationary solutions on a bounded interval, Electronic J. Diff. Eqns. 2002 (2002), no. 2, 1-12.

[13] P. Clément, L. A. Peletier, An antimaximum principle for second order elliptic operators, J. Diff. Eqns. 34 (1979), 218-229.

[14] C. Cortázar, J. Coville, M. Elgueta, S. Martínez, A non local inhomogeneous dispersal process, J. Diff. Eqns. 241 (2007), 332-358. 
[15] C. Cortázar, M. Elgueta, J.D. Rossi, A nonlocal diffusion equation whose solutions develop a free boundary, Ann. Henri Poincaré 6 (2005), 269-281.

[16] C. Cortázar, M. Elgueta, J. D. Rossi, N. Wolanski, Boundary fluxes for nonlocal diffusion, J. Diff. Eqns. 234 (2007), 360-390.

[17] C. Cortázar, M. Elgueta, J. D. Rossi, N. Wolanski, How to approximate the heat equation with Neumann boundary conditions by nonlocal diffusion problems, to appear in Arch. Rat. Mech. Anal.

[18] J. Coville, On uniqueness and monotonicity of solutions on non-local reaction diffusion equations, Ann. Mat. Pura Appl. 185 (2006), 461-485.

[19] J. Coville, Maximum principles, sliding techniques and applications to nonlocal equations, Electron. J. Diff. Eqns. 2007, no. 68 (2007), 1-23.

[20] J. Coville, J. DÁvila, S. MARTínez, Existence and uniqueness of solutions to a nonlocal equation with monostable nonlinearity, to appear in SIAM J. Math. Anal.

[21] J. Coville, L. Dupaigne, Propagation speed of travelling fronts in nonlocal reaction diffusion equations, Nonl. Anal. 60 (2005), 797-819.

[22] J. Coville, L. Dupaigne, On a nonlocal equation arising in population dynamics, Proc. Roy. Soc. Edinburgh 137 (2007), 1-29.

[23] P. Fife, Some nonclassical trends in parabolic and parabolic-like evolutions, in "Trends in nonlinear analysis", pp. 153-191, Springer-Verlag, Berlin, 2003.

[24] J. García-Melián, J. D. Rossi, On the principal eigenvalue of some nonlocal diffusion operators, preprint.

[25] J. García-Melí́n, J. SABina de LiS, Maximum and comparison principles for operators involving the p-Laplacian, J. Math. Anal. Appl. 218 (1998), 49-65.

[26] V. Hutson, S. Martínez, K. Mischaikow, G. T. Vickers, The evolution of dispersal, J. Math. Biol. 47 (2003), 483-517.

[27] L. I. Ignat, J. D. Rossi, Refined asymptotic expansions for nonlocal evolution equations, preprint.

[28] L. I. Ignat, J. D. Rossi, A nonlocal convection-diffusion equation, J. Funct. Anal. 251 (2007), 399-437.

[29] M. G. Krein, M. A. Rutman, Linear operators leaving invariant a cone in a Banach space, Amer. Math. Soc. Transl. 10 (1962), 199-325.

[30] J. LóPEz-Gómez, M. MolinA-MEyer, The maximum principle for cooperative weakly coupled elliptic systems and some applications, Diff. Int. Eqns. 7 (1994), 383-398.

[31] A. F. Pazoto, J. D. Rossi, Asymptotic behavior for a semilinear nonlocal equation, Asympt. Anal. 52 (2007), 143-155.

[32] K. Schumacher, Travelling-front solutions for integro-differential equations I, J. Reine Angew. Math. 316 (1980), 54-70.

[33] G. SweERs, Strong positivity in $C(\bar{\Omega})$ for elliptic systems, Math. Z. 209 (1992), 251-271.

[34] G. SweErs, $L^{n}$ is sharp for the antimaximum principle, J. Diff. Eqns. 134 (1997), $148-153$.

[35] W. WALter, A theorem on elliptic differential inequalities with an application to gradient bounds, Math. Z. 200 (1989), 293-299.

\section{J. GARCÍA-MELIÁN}

Dpto. de Análisis Matemático, Universidad de La Laguna.

C/. Astrofísico Francisco SÁnchez s/n, 38271 - LA Laguna, SPAin.

E-mail address: jjgarmel@ull.es

J. D. Rossi

Dpto. de Matemáticas, Facultad de Ciencias Exactas y Naturales

Universidad de Buenos Aires, 1428 - Buenos Aires, ARGEntina.

E-mail address: jrossi@dm.uba.ar 